\title{
On-chip pressure measurements and channel deformation after oil absorption
}

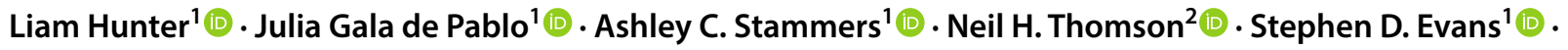 \\ Jung-uk Shim ${ }^{1}$ (1)
}

Received: 11 March 2020 / Accepted: 31 July 2020 / Published online: 11 August 2020

(c) The Author(s) 2020 OPEN

\begin{abstract}
Microfluidic channels moulded from the soft polymer poly(dimethylsiloxane) (PDMS) are widely used as a platform for mimicking biological environments, and can be used for the simulation of fluid filled structures such as blood and lung vessels. The control of pressure and flow rate within these structures is vital to mimic physiological conditions. The flexibility of PDMS leads to pressure-induced deformation under flow, leading to variable flow profiles along a device. Here, we investigate the change in Young's modulus of microfluidic channels due to infiltration of mineral oil, a PDMS permeable fluid, and how this affects the resulting pressure profile using a novel pressure measurement method. We found a 53\% decrease in Young's modulus of PDMS due to mineral oil absorption over the course of $3 \mathrm{~h}$ accounted for lower internal pressure and larger channel deformation compared to fresh PDMS at a given flow rate. Confocal fluorescence microscopy used to image channel profiles before and after the introduction of mineral oil showed a change in pressure-induced deformation after infiltration of the oil. Atomic force microscopy (AFM) nanoindentation was used to measure Young's modulus of PDMS before $(2.80 \pm 0.03 \mathrm{MPa})$ and after $(1.32 \pm 0.04 \mathrm{MPa})$ mineral oil absorption. Raman spectroscopy showed the infiltration of mineral oil into PDMS from channel walls and revealed the diffusion coefficient of mineral oil in PDMS.
\end{abstract}

Keywords Pressure drop · Channel deformation · Nanoindentation · Diffusion

\section{Introduction}

Microfluidic devices fabricated from polymers like poly(dimethylsiloxane) (PDMS) are used in a large variety of studies due to their low cost, ease of fabrication, transparency and biocompatibility [1-3]. Due to the soft nature of the polymers used, channels can be deformed under flow as a result of internal pressure build-up within the system [4-8]. While this is of use for systems such as valve structures [3], in others the change in geometry and associated pressure drops may become problematic when scaling devices. For a rigid channel, the pressure drop is

\footnotetext{
Jung-uk Shim, Liam Hunter and Stephen Evans are responsible for the study conception and design. Material preparation, data collection and analysis for confocal and pressure measurements were performed by Liam Hunter. Raman data collection and analysis was performed by Julia Gala de Pablo. AFM studies were performed by Ashley Stammers, analysed by Liam Hunter and advised upon by Neil Thomson. The first draft of the manuscript was written by Liam Hunter, and all authors commented on previous versions of the manuscript. All authors read and approved the final manuscript.
}

Electronic supplementary material The online version of this article (https://doi.org/10.1007/s42452-020-03288-8) contains supplementary material, which is available to authorised users.

Jung-uk Shim, J.Shim@leeds.ac.uk| ${ }^{1}$ Molecular and Nanoscale Physics Group, School of Physics and Astronomy, University of Leeds, Leeds LS2 9JT, UK. 'Division of Oral Biology, School of Dentistry, University of Leeds, St James's University Hospital, Leeds LS9 7TF, UK. 


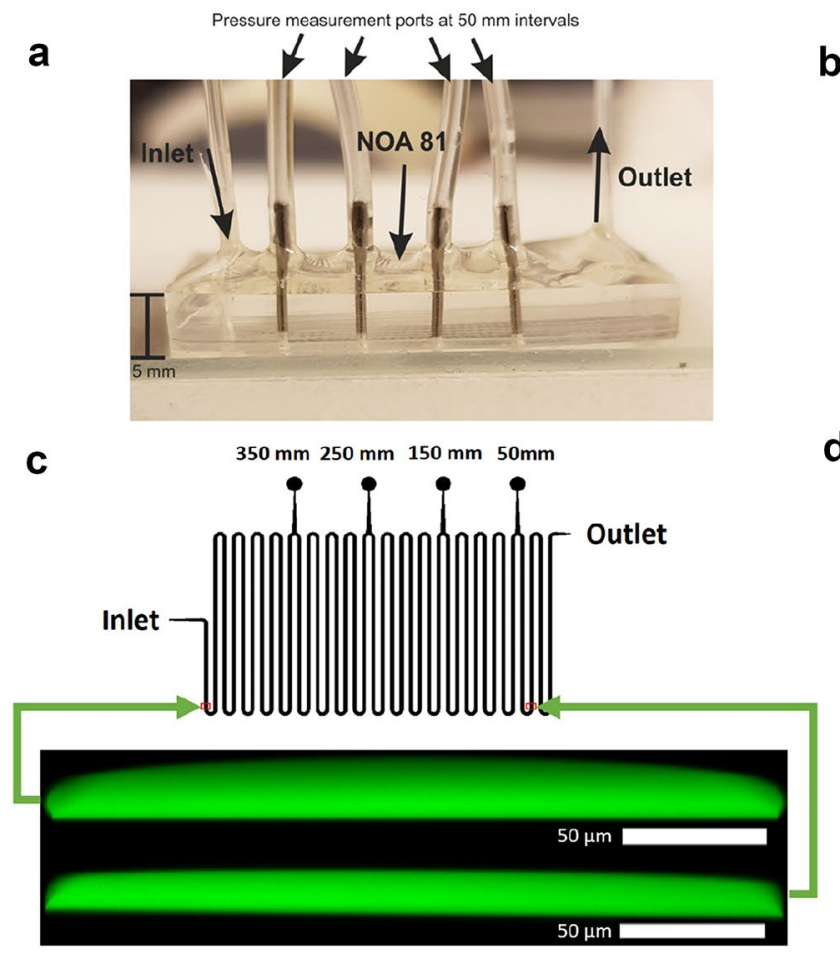

Fig. 1 a Device used in this study comprised of $250 \mu \mathrm{m}$ wide by $21 \mu \mathrm{m}$-deep channels. At $50 \mathrm{~mm}$ intervals, pressure measurement points are connected to a regulated air supply. A photograph of the device setup showing the fluid inlet and four pressure measurement ports. b A schematic representation shows the influence of pressure on a soft polymer microchannel. An increase in pressure causes the cross-sectional area normal to flow to enlarge, lowering hydraulic resistance. As a function of distance from the flow inlet, pressure and, therefore, cross-sectional area reduce before returning to the initial values of a channel under no pressure. c Confocal

proportional to the volumetric flow rate [9]. When considering a low flow rate or low pressure drop, then this proportionality holds. The constant of proportionality, the hydrodynamic resistance, is dependent on the geometry of the channel as well as the working fluids viscosity; therefore, a small channel or highly viscous liquid will increase the pressure drop. When such high pressures build-up, they lead to deformation in the channel walls [10], as illustrated in Fig. $1 \mathrm{~b}$.

As a valuable material to investigate biological systems, the deformation properties of PDMS can be tuned to match those found in vivo. This, combined with permeability to oxygen and carbon dioxide, ease of surface funtionalisation and low toxicity allows for the construction of many organ-on-a-chip-based systems [11-13]. Pressure-induced deformation of compliant microchannels has been used to model lung function through repeated actuation of a cell-covered PDMS membrane to study particle transport under differing mechanical stresses [11] or, by observing stresses and inflammatory responses, small b

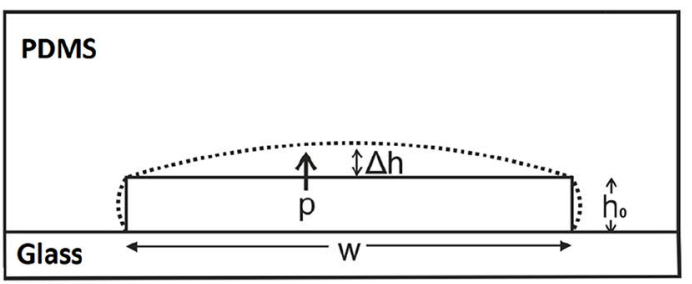

d

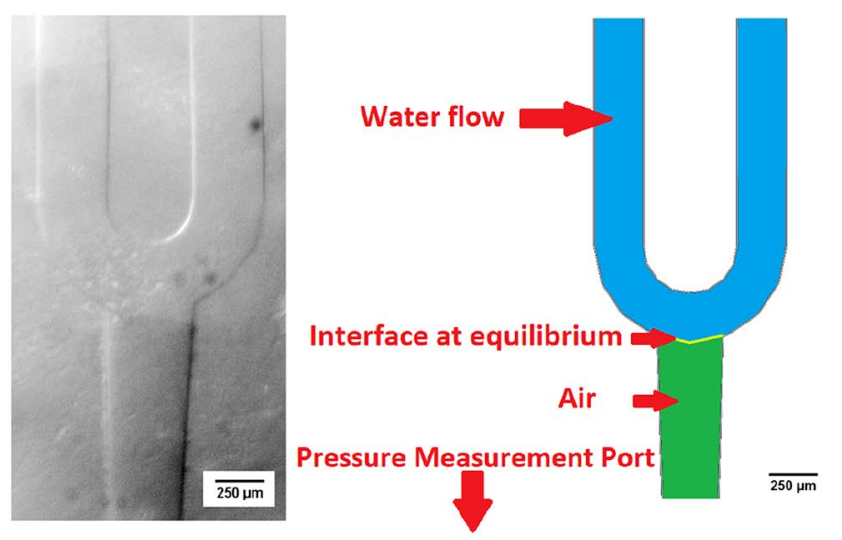

images of a channel containing fluorescein solution under a flow rate of $1800 \mu \mathrm{l} / \mathrm{h}$. Individual images taken at $z$ intervals of $0.5 \mu \mathrm{m}$ are combined to show a 2D projection of the channel profile 460 $\mathrm{mm}$ and $110 \mathrm{~mm}$ from the outlet. Under no pressure, the channel height is $21 \mu \mathrm{m}$ and under pressure of an $1800 \mu \mathrm{l} / \mathrm{h}$ flow, maximum heights of $48.41 \mu \mathrm{m}$ and $35.27 \mu \mathrm{m}$ were found, respectively. $\mathbf{d}$ The interface between injected liquid and pressurised air supply. Flow was initiated and air pressure adjusted until the interface was stable. The pressure at each measurement point could then be read

airway epithelial cells undergo when pressure drops are caused by liquid plugs rupturing in an airway channel [14]. Similar concepts have been used to model renal tubular spaces where the compliance of the channel was the key in optimising flow and shear rates for maximal cell culture [15]. The visualisation of blood flow within vessels in various states has been suggested as a viable method of studying diseased arteries. Vascular geometries have been formed with compliant PDMS channels and microvascular endothelial cells cultured under high-pressure conditions [16]. A clear understanding of the mechanical properties of the PDMS channels is required to obtain accurate measures or internal pressure and flow rates.

It is well known that Young's modulus of PDMS is an important factor to determine the deformation of the elastic polymer at a given pressure. Previous studies estimated Young's modulus of PDMS by simulating at the channel walls, with values between $950 \mathrm{kPa}$ [6] and 2.2 MPa [5]. Measurements of polymer/PDMS mechanical properties were shown in various studies [17-19] as a reliable 
method of determining PDMS modulus. Song et al. used AFM nanoindentation to measure Young's modulus of PDMS as a function of UV/Ozone treatment time showing an increase in modulus of over 100 times after 60 min of treatment. The use of AFM to measure Young's modulus has been shown to correlate with measurements of bulk systems in hydrogel polymers as well as in biological systems $[20,21]$.

Models of PDMS channel deformations have been previously developed to show the contributions of the pliable walls compared to rigid structures [5]. In this work, they combined rigid channel theory with fluidstructure interactions, stress-strain and strain-displacement to create a model for channel deformation and pressure in a PDMS channel with one channel wall being glass (assumed rigid). During the derivation of this model, assumptions were made based on the fact that the thickness of the channel ceiling was in the region of $6 \mathrm{~mm}$ and is considered a semi-infinite medium. Hardy et al. showed that the ceiling thickness greatly influences the model, whereby reduction in the thickness to $1.5 \mathrm{~mm}$ of PDMS increased the value of a proportionality constant, $\alpha$, which depends on channel geometry and mechanical properties of the channel [5]. This reduction in $\alpha$ led to an increase in channel deformability. Refinements of the model were also investigated with thin deformable channel ceilings $[4,8]$, and Raj et al. demonstrated channel deformations and pressure drops of channels with 100 and $50 \mu \mathrm{m}$ PDMS membranes as channel ceilings and could relate Gervais' fitting parameter, $\alpha$, with known membrane parameters. Christov et al. [4] further derived the model using thin membranes as channel ceilings and removed all free parameters. Cheung et al. [10] derived an expression for the deformation where the channel aspect ratio reaches unity as the Gervais model breaks down in this regime.

PDMS has also been shown to swell when exposed to certain fluids [22-24]. Lee et al. [23] tested a wide range of organic solvents and the swelling effect of the PDMS polymer network. This swelling was used to create PDMS-based solvent vapour sensors [24], investigating the swelling of channels under static flow conditions [22] through the Schlieren method where deformations in the PDMS act as a lens and distort a pattern below the channel. This work showed an increase in PDMS resistance to pressure-induced deformation with respect to crosslinker concentration in PDMS and post-crosslinking heat treatment. After this treatment, the effect of toluene on material properties was reduced and deformation was less pronounced $[25,26]$.

In this paper, we report the change Young's modulus of PDMS microchannels through absorption of mineral oil and these changes in local mechanical properties causing the internal pressure and the channel profile to differ from that of a liquid that is impermeable in PDMS. A novel pressure matching measurement method was used to obtain pressure measurements on chip. Raman spectroscopy was used to estimate a diffusion of oil in PDMS and thus understand the molecular transport in the polymer network. The Young's modulus was determined through mechanical nanoindentation with atomic force microscopy (AFM).

\section{Materials and methods}

\subsection{Device fabrication}

PDMS channels $250 \mu \mathrm{m}$ in width, $21 \mu \mathrm{m}$ in height and of a total length of $470 \mathrm{~mm}$ were fabricated using standard lithography procedures. At $100-\mathrm{mm}$ intervals, pressure measurement points were added as shown in Fig. 1. $3^{\prime \prime}$ silicon wafers (PI-KEM) were spin-coated with SU-8 2025 (MicroChem) and exposed to UV light through direct laser writing (MicroWriter ML2, Durham Magneto Optics). The wafers were silanised with trimethylchlorosilane vapour after manufacture to assist in PDMS removal after baking. PDMS pre-polymer and curing agent (Sylgard 184, Dow Corning) were mixed at a ratio of 10:1, poured onto the mould and degassed by desication. The PDMS was cured at $75^{\circ} \mathrm{C}$ for $30 \mathrm{~min}$. The PDMS was removed from the mould, inlets punched with 1 $\mathrm{mm}$ biopsy punches and sealed to number 1 thickness coverslips using $90 \mathrm{~s}$ of oxygen plasma exposure. The thickness of devices was confirmed with a surface profilometer (Dektak XT, Bruker) measuring both the SU-8 height and the PDMS channel depth.

Tubing was sealed into the device using NOA 81 optical adhesive (Norland Products) to withstand the high flow pressures, liquid delivery tubing was pushed into the inlet and outlet, and pressure measurement tubing was interfaced using cylindrical metal inserts. The metal inserts are of dimensions $330 \mu \mathrm{m}$ inner diameter and $7.6 \mathrm{~mm}$ long. This results in a pressure drop across the insert of $0.013 \mathrm{kPa}$ which is negligible compared to the pressures being measured. NOA was applied and cured under a 120-W, 365-nm UV lamp for $20 \mathrm{~min}$. The inlet tubing was made intentionally short $(100 \mathrm{~mm}$ length, $0.38 \mathrm{~mm}$ inner diameter) so that the pressure drop along it was negligible compared to the microchannel.

\subsection{On-chip pressure measurement}

Fluid was delivered to the chip using a Harvard PHD ULTRA syringe pump. The pressure measurement points 
were connected to a pressurised air supply through regulators (March Bellofram Precision Regulator, type 10). When fluid flow was initialised, the pressure regulators were adjusted to counteract flow until the gas/liquid interface was in equilibrium with the channel. Figure 1 shows a stable interface at a pressure measurement point. When all four regulators were stable, the pressure value was read. During the process of equilibrating the pressure, it was important to ensure no bubbles were present as the pressure is affected by the number of bubbles within the channel [27]. This method was repeated over multiple flow rates to generate pressure drop profiles.

To measure the effect of oil on the channel, devices were injected with mineral oil (Sigma) at a flow rate of 40 $\mu \mathrm{l} / \mathrm{h}$ for $3 \mathrm{~h}$ prior to pressure measurements. These devices were then flushed of oil using air, and then, water was flowed to measure the pressure profiles using the same method as previously described. One device was used to measure a full range of flow rates before being replaced for repeat measurements.

\subsection{Confocal channel imaging}

To determine the deformation of the channels under flow, 3D imaging was performed using a confocal microscope (Leica TCS SP8 confocal unit mounted on a DMi8 microscope) with various flow rates. Fresh PDMS devices were injected with a $1 \mu \mathrm{M}$ fluorescein solution and imaged in six locations along the length of the chip. Z-stacks were taken to obtain the height profile at each location under $40 \times$ magnification with a $z$ step size of $0.5 \mu \mathrm{m}$ Z-stacks were analysed using Image-J to obtain channel profiles and heights. Oil-saturated devices were prepared in the same way as previously described in the pressure experiments before being flushed with fluorescein solution and imaged. As with the pressure measurements, one device was used for one set of flow rate measurements.

\subsection{PDMS modulus measurements}

The modulus of PDMS films was measured using nanoindentation of the film using a Bruker FastScan AFM [18, 19]. A sharp nitride lever (SNL) probe of spring constant $0.07 \mathrm{~N} / \mathrm{m}$ was used. PDMS was probed at various locations $(n=20)$ on native PDMS and oil-saturated slabs to determine a change in Young's modulus. Each indentation was performed at least $100 \mu \mathrm{m}$ away from any other to ensure independent measurements. Fitting was performed in Bruker's NanoScope Analysis software to obtain Young's modulus values from force-displacement curves using a Sneddon conical indenter model with indenter angle $25^{\circ}$ and Poisson ratio 0.5 [28].

\subsection{Raman spectroscopy of material absorption}

For measurement of mineral oil diffusion into PDMS, devices were prepared on $60 \times 24 \mathrm{~mm}$ coverslips of \# 1 thickness (130-160 $\mu \mathrm{m})$. The Raman system used a $532 \mathrm{~nm}$ laser as an excitation source from an inVia Raman confocal inverted microscope (Renishaw) with light collected using a Newton EMCCD sensor (DU970P, Andor, $1600 \mathrm{X}$ $200 \mathrm{px}$ ). A silicon sample was used to calibrate to the peak position of $520.5 \mathrm{~cm}^{-1}$ prior to each experiment. Raman spectra were taken of pure mineral oil, pure PDMS and an empty channel to identify characteristic peaks as shown in figure S1. A scan line of length $1 \mathrm{~mm}$ was positioned so that it began in the empty channel and ended within the bulk PDMS. Along this line, spectra were taken every $20 \mu \mathrm{m}$ with an acquisition time of $1 \mathrm{~s}$. After this scan, the injection of mineral oil was started and spectra were taken along the scan line every $5 \mathrm{~min}$ for $90 \mathrm{~min}$.

\section{Results and discussion}

The pressure measured along the microchannel before oil infiltration into PDMS, at 4 points as a function of fluid flow rate, $Q$, of water and the resulting channel deformation is shown in Fig. 2. The pressure increases as a function of channel position from the outlet and is not linear, as might be expected for non-compliant microchannels of the same rectangular geometry [5]. The internal pressure of the microchannels increased as a function of flow rate with maximum pressures measured, at the point closest to the inlet of $149 \pm 8 \mathrm{kPa}$ and $278 \pm 9 \mathrm{kPa}$ for flow rates of $600 \mu \mathrm{l} / \mathrm{h}$ and $1800 \mu \mathrm{l} / \mathrm{h}$, respectively. Above $1800 \mu \mathrm{l} / \mathrm{h}$, the pressure inside the channel causes NOA 81 to fail and leak. To calculate theoretical channel pressure values, the model derived by Gervais et al. was used and is given in Eq. 1 . The theoretical pressures were calculated using MATLAB to solve the equation for $p(z)$ (Eq. 2) at each flow rate using Young's modulus values measured by AFM nanoindentation. Measurement of Young's modulus, $E$, on fresh PDMS with no modification yielded a value of $2.80 \pm 0.03 \mathrm{MPa}$, which is quite similar to modulus reported by Song et al. [18]. Fitting the data to the theoretical values showed that for this system, the proportionality constant $\alpha=0.7$ which is in line with Gervais' predictions that $\alpha$ be of order 1.

$$
Q=\frac{h_{0}^{4} E}{48 \alpha \mu(L-z)}\left[\left(1+\alpha \frac{p(z) W}{E h_{0}}\right)^{4}+1\right]
$$

Solved for $p(z)$ : 
a

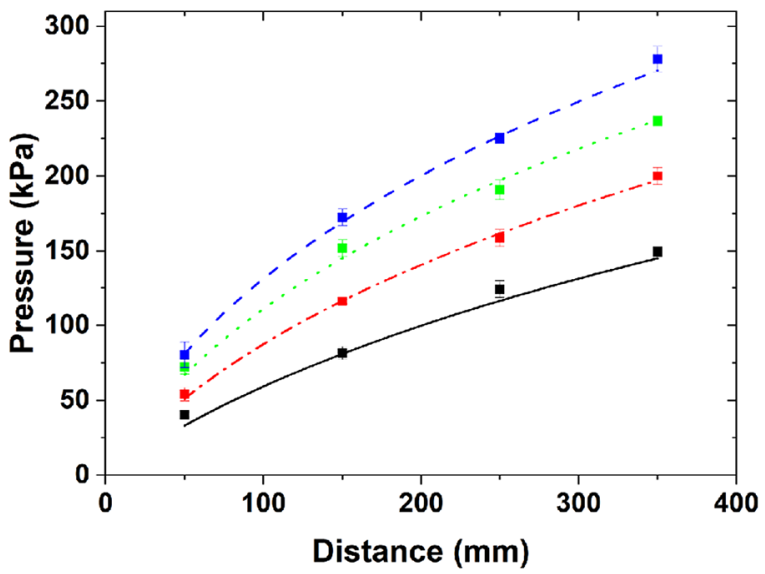

C

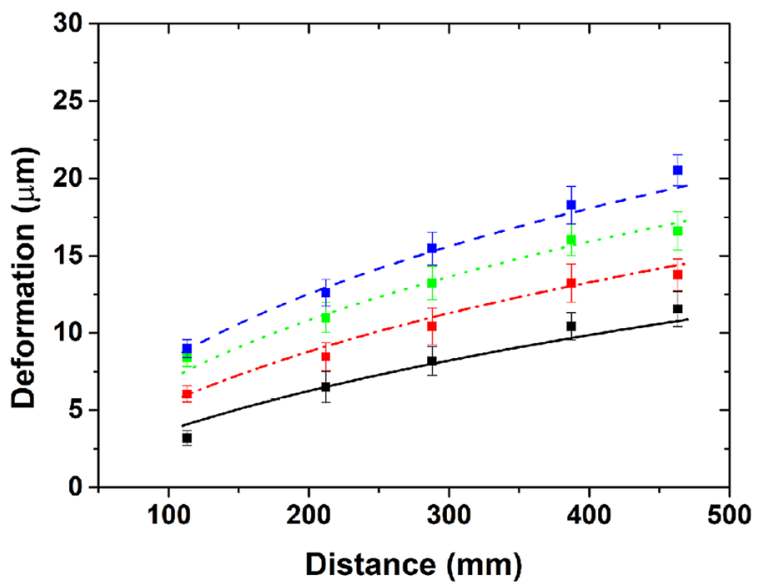

Fig. 2 Pressure drop as a function of distance from the outlet along a PDMS microchannel before (a) and after (b) $3 \mathrm{~h}$ soaking with mineral oil measured with pressure matching measurement ports. Coloured lines show calculated theoretical values using Eq. 1 with $E=(2.80 \pm 0.03) \mathrm{MPa}$ in (a) and $(1.32 \pm 0.04) \mathrm{MPa}$ in (b) where black, red, green and blue are equal to $600,1000,1400$ and 1800

$p(z)=-\frac{E h-2 \sqrt{6 E^{3} \sqrt{\frac{h_{0}^{4}}{576 E^{2}}+\frac{L \alpha Q \mu}{12 E^{3}}-\frac{\alpha Q \mu z}{12 E^{3}}}}}{W \alpha}$

where $Q=$ fluid flow rate, $h_{0}=$ channel initial height, $E=$ Young's modulus, $\alpha=$ fitting parameter, $\mu=$ fluid viscosity, $L=$ channel length, $z=$ channel position, $p(z)=$ channel internal pressure and $W=$ channel width.

Deformation of the channels was investigated using a confocal microscope by flowing a fluorescein solution in the channel and the resulting height profiles in Fig. 2 show an increase in channel height as the flow rate increases as expected. Close to the outlet and near the inlet, shown in Fig. 1, defines the scale of deformation within the channel. Ceiling deformation at positions $460 \mathrm{~mm}$ and $110 \mathrm{~mm}$ from the outlet is 21 and $9 \mu \mathrm{m}$, respectively, a $43-100 \%$ increase. The z-stacks were analysed using ImageJ to obtain the

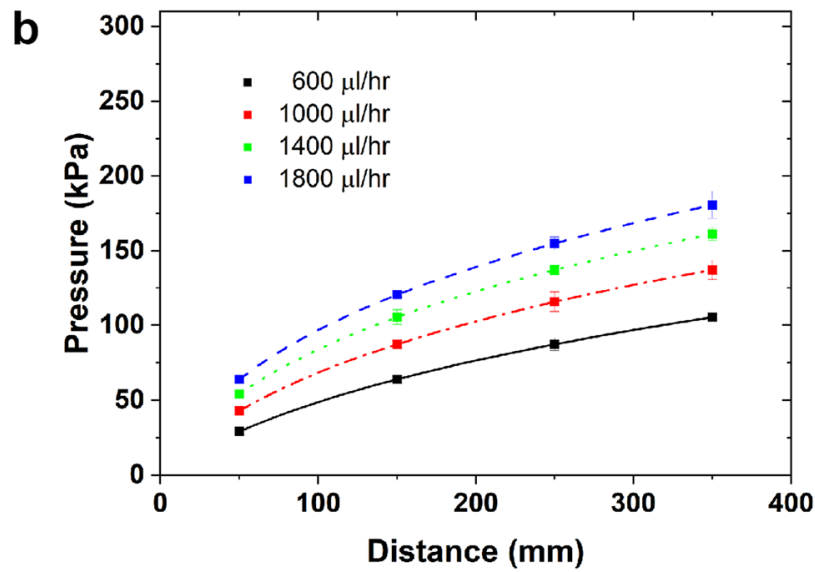

d

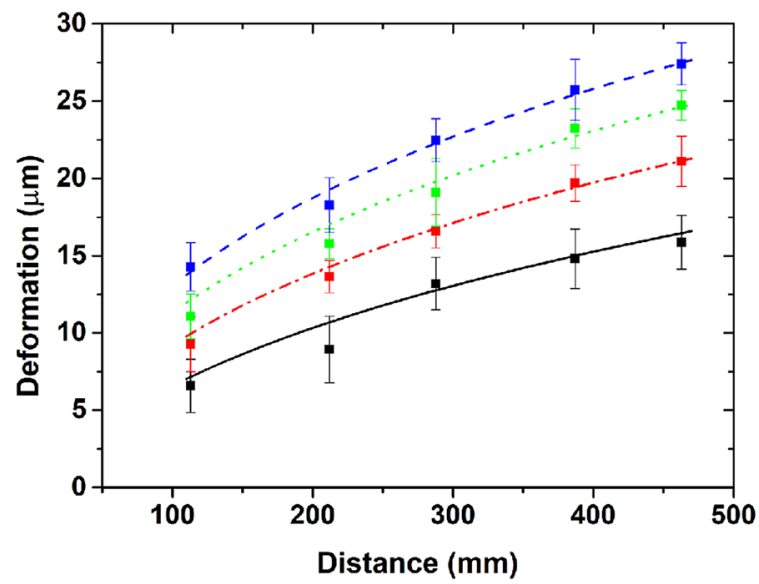

$\mu \mathrm{l} / \mathrm{h}$, respectively. Deformation of channel height as a function of distance from the outlet along the microchannel $\mathbf{c}$ before soaking and $\mathbf{d}$ after, measured using confocal microscopy with solid lines of theoretical values from Eq. 3 using Young's modulus values previously described

profile and height of the channel. As shown in Fig. 2, there is a deformation of the side-channel walls but is negligible compared to the ceiling deformation in agreement with Gervais et al. [5]. Values for the predicted height change were obtained by substituting previously calculated pressure values into Eq. 3 where $h(z)$ is the deformed channel height.

$h(z)=h_{0}\left(1+\alpha \frac{p(z) W}{E h_{0}}\right)$

The pressure was measured for a channel that had previously had mineral oil flowing through at a rate of $40 \mu$ $\mathrm{I} / \mathrm{h}$ for $3 \mathrm{~h}$ to test if the absorption of oil into the PDMS was changing the response of the polymer to the applied pressure. Measurements showed a decrease in the internal channel pressure when compared to a channel of fresh PDMS. The resulting pressure profiles in Fig. 2 show that at 
the same flow rates, the pressure was reduced indicating an increase in channel cross section. The data in Fig. 2 were compared to theoretical pressure values calculated using Young's modulus of oil-absorbed PDMS measured through AFM nanoindentation. The nanoindentation showed that after exposure to mineral oil and its subsequent absorption, the Young's modulus changed to $E=(1.32 \pm 0.04)$ $\mathrm{MPa}$, a reduction of $53 \%$. Using this reduced modulus, the theoretical values agree with the measured values while also maintaining proportionality constant $\alpha=0.7$.

Raman spectroscopy was used to show the depth of penetration of mineral oil into PDMS when flowed through a microchannel. The spectra of pure mineral oil and PDMS were taken and are shown in figure S1. The PDMS peak at $709 \mathrm{~cm}^{-1}$ was used for normalisation of all spectra, and the mineral oil peak at $2849 \mathrm{~cm}^{-1}$ was integrated and plotted against time and distance in figure $\mathrm{S} 3$. The white dashed line in the figure shows the edge of the channel, and it can be seen that the mineral oil is diffusing into the PDMS over time. The data were fitted to calculate the diffusion coefficient, $D$, of mineral oil into the PDMS. This was modelled as a one-dimensional diffusion with a constant reservoir of material available. The Raman intensity as a function of distance at various times is shown in Fig. 3. The intensities of Raman signal increased as time progresses, and the solid lines shown are fitting of a one-dimensional diffusion. From these diffusion profiles, a diffusion coefficient $D=(4.7 \pm 0.4) \times 10^{-12} \mathrm{~m}^{2} \mathrm{~s}^{-1}$ was obtained. This result is comparable to diffusion coefficients obtained for similar solvents measured by Dangala et al. through bulk swelling experiments [22].

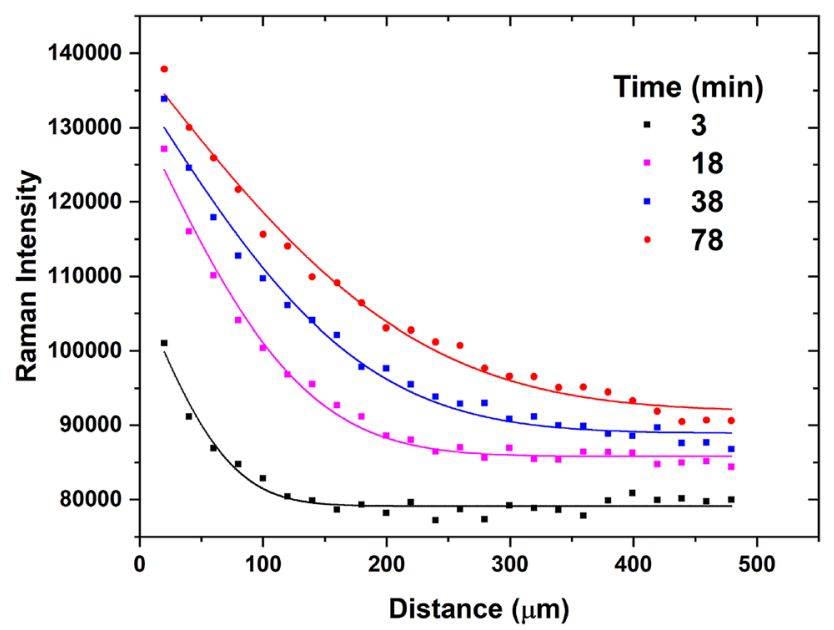

Fig. 3 Raman intensity versus distance away from the microfluidic channel shows the infiltration of mineral oil into PDMS. As a function of time, the Raman signal increases indicating permeation of oil into the bulk polymer. The solid lines show theoretical values calculated through a one-dimensional Fickian diffusion model

\section{Conclusions}

The Young's modulus of the walls of a PDMS microchannel is the key in determining the pressure drop profile and deformation of the channel under flow. The diffusion of oil into the walls of a channel vastly decreases the modulus causing increased deformation under the same pressure. The diffusion of mineral oil into the PDMS polymer matrix over a period of $3 \mathrm{~h}$ was shown to reduce Young's modulus by $53 \%$ when measured through AFM nanoindentation. This significant change in fluid flow profile in the channel over time could interfere with pressure sensitive experiments, for example the growth of cells in a biological mimicked system. As cells can grow on the scale of days, the presence of a PDMS soluble fluid will greatly interfere with the initial flow profiles.

Raman spectroscopy showed the permeation of mineral oil into PDMS when flowed through a microchannel and indicated that the oil was absorbed sufficiently far into the polymer matrix to change the effective Young's modulus. The diffusion coefficient of mineral oil into PDMS was obtained using a one-dimensional Fickian diffusion model and agrees with previous studies of hydrocarbon oil absorption in to PDMS polymer [22].

A method for measuring pressures in the microchannels on-chip was developed using a regulated air supply to balance internal pressure exhibited by fluid flows. The 4-point measurement along the channel with measurements of Young's modulus of PDMS shows that this technique provides an accurate measurement of pressure that agrees with current models derived for internal pressures of thick-walled PDMS microchannels. The channel profile was measured through devices where the channel walls had been soaked with mineral oil showing an increase in the wall deformity compared to a fresh device under the same flow conditions leading to lower internal pressures. Confocal microscopy of the channels showed the increase in channel cross-sectional area leading to the reduced pressures that were measured.

Although previous studies showed methods to improve resistance against pressure-induced deformation and swelling $[25,26]$, the change in material properties due to the solvent absorption is an important factor to be considered when designing applications with PDMS devices. This work shows that the introduction of fluids during experimentation can modify the local PDMS properties and change the pressure distribution along a channel. This may be a benefit as devices become larger and more complex where high back pressures could become destructive, especially for soft polymer devices. The increasing research into biological systems using microfluidic devices requires careful considerations of material choices when designing 
vessels where fluid structure interactions are being observed. The time-dependent change in local material properties can skew observations, and maintaining constant parameters is essential for realistic in vitro studies.

Datasets for this paper are publically available in the University of Leeds Data Repository (https://doi. org/10.5518/737)

Acknowledgements The authors acknowledge the financial support of the Engineering and Physical Sciences Research Council (Grant No. EP/M506552/1).

\section{Compliance with ethical standards}

Conflict of interest The authors declare that they have no competing interests.

Open Access This article is licensed under a Creative Commons Attribution 4.0 International License, which permits use, sharing, adaptation, distribution and reproduction in any medium or format, as long as you give appropriate credit to the original author(s) and the source, provide a link to the Creative Commons licence, and indicate if changes were made. The images or other third party material in this article are included in the article's Creative Commons licence, unless indicated otherwise in a credit line to the material. If material is not included in the article's Creative Commons licence and your intended use is not permitted by statutory regulation or exceeds the permitted use, you will need to obtain permission directly from the copyright holder. To view a copy of this licence, visit http://creativecommons .org/licenses/by/4.0/.

\section{References}

1. McDonald JC, Duffy DC, Anderson JR, Chiu DT, Wu H, Schueller OJA, Whitesides GM (2000) Fabrication of microfluidic systems in poly(dimethylsiloxane). Electrophoresis 21(1):27-40. https://doi. org/10.1002/(sici)1522-2683(20000101)21:1\%3C27::aid-elps2 7\%3E3.0.co;2-c

2. Raj A, Suthanthiraraj PPA, Sen AK (2018) Pressure-driven flow through PDMSbased flexible microchannels and their applications in microfluidics. Microfluid Nanofluidics 22(11):128. https:// doi.org/10.1007/s10404-018-2150-5

3. Unger MA (2000) Monolithic microfabricated valves and pumps by multilayer soft lithography. Science 288(5463):113-116. https ://doi.org/10.1126/science.288.5463.113

4. Christov IC, Cognet V, Shidhore TC, Stone HA (2018) Flow rate-pressure drop relation for deformable shallow microfluidic channels. J Fluid Mech 841:267-286. https://doi.org/10.1017/jfm.2018.30. arXiv: 1712.02687

5. Gervais T, El-Ali J, Günther A, Jensen KF (2006) Flow-induced deformation of shallow microfluidic channels. Lab Chip 6(4):500-507. https://doi.org/10.1039/b513524a

6. Hardy BS, Uechi K, Zhen J, Pirouz Kavehpour H (2009) The deformation of flexible PDMS microchannels under a pressure driven flow. Lab Chip 9(7):935-938. https://doi.org/10.1039/b813061b

7. Raj A, Sen AK (2016) Flow-induced deformation of compliant microchannels and its effect on pressure-flow characteristics. Microfluid Nanofluidics 20(2):1-13. https://doi.org/10.1007/s1040 4-016-1702-9

8. Raj MK, DasGupta S, Chakraborty S (2017) Hydrodynamics in deformable microchannels. Microfluid Nanofluidics 21(4):1-12. https://doi.org/10.1007/s10404-017-1908-5
9. Bruus $\mathrm{H}$ (2008) Theoretical microfluidics. Oxford university press, Oxford. https://doi.org/10.1111/j.1574-6968.2009.01808.x. arXiv: arXiv:1011.1669v3

10. Cheung P, Toda-Peters K, Shen AQ (2012) In situ pressure measurement within deformable rectangular polydimethylsiloxane microfluidic devices. Biomicrofluidics 6(2):026501. https://doi. org/10.1063/1.4720394

11. Huh D, Matthews BD, Mammoto A, Montoya-Zavala M, Yuan Hsin $\mathrm{H}$, Ingber DE (2010) Reconstituting organ-level lung functions on a chip. Science 328(5986):1662-1668. https://doi.org/10.1126/scien ce. 1188302

12. Kim S, Takayama S (2015) Organ-on-a-chip and the kidney. Kidney Res Clin Pract 34(3):165-169. https://doi.org/10.1016/j. krcp.2015.08.001

13. Zhang B, Korolj A, Lai BFL, Radisic M (2018) Advances in organon-a-chip engineering. Nat Rev Mater 3(8):257-278. https://doi. org/10.1038/s41578-018-0034-7

14. Huh D, Fujioka H, Tung YC, Futai N, Paine R, Grotberg JB, Takayama $S$ (2007) Acoustically detectable cellular-level lung injury induced by fluid mechanical stresses in microfluidic airway systems. Proc Natl Acad Sci USA 104(48):18886-18891. https://doi.org/10.1073/ pnas.0610868104

15. Jang KJ, Suh KY (2010) A multi-layer microfluidic device for efficient culture and analysis of renal tubular cells. Lab Chip 10(1):3642. https://doi.org/10.1039/b907515a

16. Shin M, Matsuda K, Ishii O, Terai H, Kaazempur-Mofrad M, Borenstein J, Vacanti JP (2004) Endothelialized networks with a vascular geometry in microfabricated poly(dimethyl siloxane). Biomed Microdevices 6(4):269-278. https://doi.org/10.1023/ B:BMMD.0000048559.29932.27

17. Johnston ID, McCluskey DK, Tan CK, Tracey MC (2014) Mechanical characterization of bulk Sylgard 184 for microfluidics and microengineering. J Micromech Microeng 24(3):035017. https://doi. org/10.1088/0960-1317/24/3/035017

18. Song J, Tranchida D, Vancso GJ (2008) Contact mechanics of UV/ ozone-treated PDMS by AFM and JKR testing: mechanical performance from nano- to micro-meter length scales. Macromolecules 41(18):6757-6762. https://doi.org/10.1021/ma800536y

19. Tranchida D, Piccarolo S, Soliman M (2006) Nanoscale mechanical characterization of polymers by AFM nanoindentations: critical approach to the elastic characterization. Macromolecules 39(13):4547-4556. https://doi.org/10.1021/ma052727j

20. Engler AJ, Rehfeldt F, Sen S, Discher DE (2007) Microtissue elasticity: measurements by atomic force microscopy and its influence on cell differentiation. Methods Cell Biol 83(07):521-545. https:// doi.org/10.1016/S0091-679X(07)83022-6

21. Tronci G, Grant CA, Thomson NH, Russell SJ, Wood DJ (2015) Multi-scale mechanical characterization of highly swollen photoactivated collagen hydrogels. J R Soc Interface 20141079. https:// doi.org/10.1098/rsif.2014.1079

22. Dangla R, Gallaire F, Baroud CN (2010) Microchannel deformations due to solvent induced PDMS swelling. Lab Chip 10(21):29722978. https://doi.org/10.1039/c003504a

23. Lee JN, Park C, Whitesides GM (2003) Solvent compatibility of poly(dimethylsiloxane)—based microfluidic devices. Anal Chem 75(23):6544-6554. https://doi.org/10.1021/ac0346712

24. Rumens CV, Ziai MA, Belsey KE, Batchelor JC, Holder SJ (2015) Swelling of PDMS networks in solvent vapours; applications for passive RFID wireless sensors. J Mater Chem C 3(39):10091-10098. https://doi.org/10.1039/C5TC01927C

25. Cesaria M, Arima V, Manera MG, Rella R (2018) Protocol of thermal aging against the swelling of poly(dimethylsiloxane) and physical insight in swelling regimes. Polymer (United Kingdom) 139:145154. https://doi.org/10.1016/j.polymer.2018.02.022

26. Kim M, Huang Y, Choi K, Hidrovo CH (2014) The improved resistance of PDMS to pressure-induced deformation and chemical 
solvent swelling for microfluidic devices. Microelectron Eng 124:66-75. https://doi.org/10.1016/j.mee.2014.04.041

27. Fuerstman MJ, Lai A, Thurlow ME, Shevkoplyas SS, Stone HA, Whitesides GM (2007) The pressure drop along rectangular microchannels containing bubbles. Lab Chip 7(11):1479-1489. https:// doi.org/10.1039/b706549c

28. Müller A, Wapler MC, Wallrabe U (2019) A quick and accurate method to determine the Poisson's ratio and the coefficient of thermal expansion of PDMS. Soft Matter 15(4):779-784. https:// doi.org/10.1039/c8sm02105h

Publisher's Note Springer Nature remains neutral with regard to jurisdictional claims in published maps and institutional affiliations. 